\title{
ATENÇÃO FARMACÊUTICA AO CUIDADOR DE PACIENTE COM DOENÇA DE ALZHEIMER*
}

Geraci Oliveira Santos ${ }^{1}$, Cláudia Zamberlan², Jane Beatriz Limberger ${ }^{3}$

\begin{abstract}
RESUMO: Objetivou-se por meio deste estudo avaliar as necessidades de saúde de cuidadores de pacientes com Doença de Alzheimer no que se refere ao autocuidado, identificando ocasiões favoráveis e propondo estratégias de intervenção farmacêutica para a melhoria da qualidade de vida. O estudo caracteriza-se como descritivo e exploratório pelo método observacional. A coleta de dados foi conduzida por meio da aplicação de questionário a 83 cuidadores, após a aprovação por comitê de ética em pesquisa. Observou-se que os cuidadores eram, em sua maioria, mulheres, casadas, com filhos, com média de idade de 38,93 \pm 11,42 anos, não praticantes de atividade física, com sobrecarga de atividades, gerando demandas em saúde e oportunidades de atuação no cuidado farmacêutico. O desenvolvimento de ações e/ou intervenções que contemplem as necessidades observadas favorece a qualidade de vida dos indivíduos e contribui com a assistência em saúde.
\end{abstract}

DESCRITORES: Doença de Alzheimer; Cuidadores; Atenção farmacêutica.

\section{PHARMACEUTICAL CARE FOR THE CAREGIVER OF THE PATIENT WITH ALZHEIMER'S DISEASE}

\begin{abstract}
This study's aim was to evaluate the health needs of caregivers of patients with Alzheimer's Disease regarding self-care, identifying favorable occasions and proposing pharmaceutical intervention strategies for improving their quality of life. The study is characterized as descriptive and exploratory, using the observational method. Data collection occurred by applying a questionnaire to 83 caregivers, after the approval of the Research Ethics Committee. It was observed that the majority of the caregivers were women, married, with children, with a mean age of $38.93 \pm 11.42$ years old, not practising physical activity and overloaded with activities, creating health demands and opportunities for receiving pharmaceutical care. The undertaking of actions and/or interventions which cover the needs observed favors the individuals' quality of life and contributes to the health care. DESCRIPTORS: Alzheimer's Disease; Caregivers; Pharmaceutical care.
\end{abstract}

\section{ATENCIÓN FARMACÉUTICA AL CUIDADOR DE PACIENTE CON ALZHEIMER}

RESUMEN: El objetivo de este estudio fue evaluar las necesidades de salud de cuidadores de pacientes con enfermedad de Alzheimer acerca del autocuidado, identificando ocasiones favorables y proponiendo estrategias de intervención farmacéutica para mejorar la cualidad de vida. El estudio se caracteriza como descriptivo y exploratorio por el método observacional. Los datos fueron recogidos por medio de la aplicación de cuestionario a 83 cuidadores, después de la aprobación por comité de ética en investigación. Se observó que los cuidadores eran, en mayoría, mujeres, casadas, con hijos, con media de edad de 38,93 \pm 11,42 años, no practicantes de actividad física, con sobrecarga de actividades, generando demandas en salud y oportunidades de actuación en el cuidado farmacéutico. El desarrollo de acciones y/o intervenciones que contemplen las necesidades observadas favorece la cualidad de vida de los individuos y contribuye con la asistencia en salud.

DESCRIPTORES: Enfermedad de Alzheimer; Cuidadores; Atención farmacéutica.

\footnotetext{
*Trabalho final de graduação apresentado ao Curso de Farmácia do Centro Universitário Franciscano - UNIFRA em 2012.

${ }^{1}$ Farmacêutica.

${ }^{2}$ Enfermeira. Mestre em Enfermagem. Doutoranda em Enfermagem pela Universidade Federal do Rio Grande. Professora do UNIFRA. Membro do Grupo Interdisciplinar de Pesquisa em Educação e Saúde - GIPES.

${ }^{3}$ Farmacêutica Industrial. Mestre em Química. Coordenadora e Professora do Curso de Graduação em Farmácia do UNIFRA. Membro do Grupo de Pesquisas em Ciências Farmacêuticas.
}

Autor correspondente:

Jane Beatriz Limberger

Centro Universitário Franciscano

Rua dos Andradas, 1614 - 97010-032 - Santa Maria-RS-Brasil

E-mail: janebeatriz@unifra.br
Recebido: 01/03/2013

Aprovado: 28/08/2013 


\section{INTRODUÇÃO}

A população, tanto no Brasil quanto no mundo, está mais velha, sendo este fato resultante da diminuição das taxas de mortalidade e da maior expectativa de vida e melhora das condições de saúde ${ }^{(1)}$. O envelhecimento é definido como a redução da capacidade funcional após o período de maturidade reprodutiva, sendo fisiologicamente mais uma das etapas da vida dos seres vivos. Essa etapa da vida determina uma maior incidência de doenças crônicas neurodegenerativas, sendo a mais frequente a Doença de Alzheimer (DA), patologia neurológica progressiva e irreversível, marcada por perdas graduais da função cognitiva e distúrbios do comportamento e afeto. Essa doença afeta o cérebro produzindo atrofia, determinando a perda das habilidades de pensar, raciocinar, memorizar, comprometendo áreas da linguagem e produzindo as alterações no comportamento ${ }^{(2)}$.

Ao saber do diagnóstico e das implicações da DA, muitas pessoas, tais como profissionais e familiares, dedicam-se, quase que exclusivamente, ao cuidado do paciente, por vezes abrindo mão da vida social e afetiva. Estudos demonstram que cuidadores que se dedicam sozinhos a esta atividade tem risco aumentado de desenvolver depressão, ansiedade e distúrbios de imunidade ${ }^{(3)}$. Por este motivo, além dos cuidados com o paciente deve-se atentar também ao cuidador, devido à sobrecarga física e emocional. Tal situação compromete sua qualidade de vida, pois o cuidado a um adulto dependente envolve o desgaste e ambivalência entre cuidar dos outros e descuidar-se, no limiar entre razão e emoção ${ }^{(1)}$.

Por outro lado, considera-se como cuidador não só a pessoa com grau de parentesco ou de amizade e vizinhança com o paciente, mas também todo indivíduo que pratica o ato de cuidar em diferentes momentos e situações distintas de suas vidas, podendo este fazer parte de uma equipe de saúde ${ }^{(4)}$. Um cuidador pode apresentar desgaste físico e mental quando exclusivamente dedicado ao ofício e sem receber ajuda, muitas vezes sendo incumbido deste papel devido à circunstância, e não por escolha própria. Esse cuidador familiar não antevê o quanto lhe será exigido, mas, se a estrutura familiar for favorável, será melhor para todos, principalmente para quem desenvolve a doença ${ }^{(3)}$.

Como ainda é desconhecida a cura da DA, e havendo poucas opções de tratamento, se faz imprescindível os cuidados que, mesmo paliativos, são necessários ao conforto do paciente e seus familiares. E, se o cuidador familiar não se encontrar em boas condições de saúde, devido às exigências que esta tarefa requer, a saúde e o cuidado do paciente acometido também estarão comprometidos.

Portanto, conhecer a evolução dos sintomas da DA com a permanente avaliação da vulnerabilidade do idoso e seu cuidador/familiar tem relevância na preparação de estratégias para prevenir as possíveis dificuldades, proporcionando a melhoria na qualidade de vida do paciente e sua família ${ }^{(5)}$. Assim, objetivou-se, por meio deste estudo, conhecer os aspectos de saúde relacionados a hábitos de consumo de medicamentos e autocuidado em cuidadores de pacientes com DA, promovendo a atenção farmacêutica por meio de intervenções farmacoterapêuticas, na busca da resolução de problemas relacionados a medicamentos, melhoria da adesão e da qualidade de vida destes usuários.

\section{MÉTODO}

O estudo caracteriza-se como descritivo e exploratório pelo método observacional. A pesquisa descritiva tem por objetivo descrever as características de uma determinada população ou fenômeno, estabelecendo relações entre as variáveis. Já a exploratória teve como objetivo principal aprimorar ideias ou a descoberta de intuições ${ }^{(6)}$. A coleta de dados foi conduzida por meio da aplicação de um questionário destinado a 83 cuidadores de pacientes com DA, sendo preservadas sua identidade, com aprovação da Comissão de Pesquisa e Ética em Saúde do Centro Universitário Franciscano, sendo registrada na Plataforma Brasil sob o número CAAE 05565212.1.0000.5306.

Fez parte do estudo um grupo inicial de cuidadores que frequenta o Grupo Assistência Multidisciplinar Integrada aos Cuidadores dos Portadores da Doença de Alzheimer - AMICA, sem distinção de idade ou sexo. Ao grupo inicial, composto por 10 cuidadores e denominado "primeira geração", foi solicitado que indicassem outros cuidadores com a mesma condição para também fazer parte da pesquisa. Nesta "segunda geração" obteve-se um grupo de 28 indivíduos, para os quais também foi solicitada a indicação de outros cuidadores, quando se obteve a "terceira geração", composta por mais 45 indivíduos, perfazendo o total de 83 participantes. A seleção dos participantes, realizada por meio da metodologia denominada bola de neve ${ }^{(7)}$, respeitou os critérios de inclusão da amostra, ou seja, o princípio da aleatoriedade, do voluntariado e a atuação como cuidador de paciente com DA, tanto 
para familiar ou uma pessoa assim reconhecida, quanto cuidador remunerado exercendo o papel de cuidador por, no mínimo, um mês. Os dados foram coletados nos meses de setembro e outubro de 2012.

A amostra foi não probabilística e por método de conveniência, sendo convidados os cuidadores que aceitassem participar do estudo, que atendessem aos critérios de inclusão, assinando o Termo de Consentimento Livre e Esclarecido. Foram observados os aspectos éticos, atendendo à Resolução 196/96 do Conselho Nacional de Saúde, com a finalidade de seguir as normas éticas envolvendo pesquisa com seres humanos. Foi assegurado o sigilo e os cuidadores foram informados quanto aos objetivos da pesquisa.

\section{RESULTADOS}

Na Tabela 1 estão listadas as principais características sociodemográficas relacionadas aos cuidadores de pacientes com DA, 22,9\% tinham entre 20 e 29 anos; $24,1 \%$ entre 30 a 39 anos; $31,3 \%$ entre 40 a 49 anos; $16,9 \%$ entre 50 a 59 anos e $4,8 \%$ estavam na faixa etária entre 60 a 69 anos. Entre os que referiram realizar atividade física, $55,5 \%$ praticam caminhada, $5,5 \%$ corrida, $5,5 \%$ futebol, $11,1 \%$ natação e $22,4 \%$ frequentam academia. Entre os que referiram hábito de beber, $80 \%$ ingeriam bebida duas vezes por semana e $20 \%$ três 3 vezes por semana.

Ao serem questionados sobre quantas horas diárias trabalham, boa parte declarou exercer a atividade de cuidador por seis ou 12 horas diárias com idênticos per- centuais, de 34,9\%; em relação ao repouso, a maioria referiu dormir seis e oito horas diariamente $(42,2 \%$ e $41,0 \%$, respectivamente). Com relação ao grau de parentesco do entrevistado com o portador de DA, 90,4\% não possuem parentesco; 7,2\% são filhos e $2,4 \%$ têm outros graus de parentesco; e $8,5 \%$ coabitavam com o paciente e $91,5 \%$ não coabitavam.

Quando perguntados se apresentam algum problema de saúde, $73,5 \%$ responderam que não possuíam, enquanto que $26,5 \%$ responderam que sim. Destes, a metade apresenta hipertensão, 9,4\% depressão; 9,4\% dores musculares; e com percentuais idênticos referiram possuir diabetes, enfisema pulmonar, problemas na coluna cervical, ansiedade, varizes, problemas auditivos e hipoglicemia (7,2\%).

Ao serem questionados se o problema de saúde interfere em suas atividades diárias, 21,6\% responderam que sim e 78,3\% que não interfere no seu dia-a-dia. Quanto à utilização de medicamentos, 68,7\% responderam que não usavam, enquanto que $31,3 \%$ responderam que sim; entre esses, $46,1 \%$ tomavam o medicamento uma vez ao dia; $30,7 \%$ duas vezes ao dia; $15,3 \%$ três vezes ao dia e $7,9 \%$ quatro vezes ao dia. Aos que faziam uso de medicamentos 10,9\% encontram alguma dificuldade na sua utilização. Das dificuldades apontadas, $77,8 \%$ esquecem de tomar o medicamento e $22,2 \%$ encontram dificuldades com os efeitos colaterais apresentados. Em relação aos exames médicos de rotina, $66,3 \%$ responderam que realizam uma visita ao médico por ano.

Em relação a tratamento ou acompanhamento com

Tabela 1 - Características gerais dos cuidadores de pacientes com DA. Santa Maria, 2012

\begin{tabular}{lcclcc}
\hline Idade & 38,93 (média) $\pm 11,42(\mathrm{DP} \%)$ & & $\mathbf{N}$ & $\mathbf{\%}$ \\
\hline Sexo & $\mathbf{N}$ & $\mathbf{\%}$ & & 71 & 85,5 \\
Masculino & 12 & 14,5 & Feminino & $\mathbf{N}$ & $\mathbf{\%}$ \\
\hline Situação conjugal & $\mathbf{N}$ & $\mathbf{\%}$ & & 12 & 14,5 \\
Casado & 40 & 48,2 & Separado & 6 & 7,2 \\
Solteiro & 25 & 30,1 & Outro & $\mathbf{N}$ & $\mathbf{\%}$ \\
\hline Filhos & $\mathbf{N}$ & $\mathbf{\%}$ & & 29 & 34,4 \\
Sim & 54 & 65,6 & Não & $\mathbf{N}$ & $\mathbf{\%}$ \\
\hline Atividade física & $\mathbf{N}$ & $\mathbf{\%}$ & & 47 & 56,3 \\
Sim & 36 & 43,4 & Não & $\mathbf{N}$ & $\mathbf{\%}$ \\
\hline Tabagismo & $\mathbf{N}$ & $\mathbf{\%}$ & & 72 & 86,7 \\
Sim & 11 & 13,3 & Não & $\mathbf{N}$ & $\mathbf{\%}$ \\
\hline Consumo de álcool & $\mathbf{N}$ & $\mathbf{\%}$ & & $\mathbf{N}$ & 82,0 \\
Sim & 15 & 18,0 & Não & 15 & $\mathbf{\%}$ \\
\hline Turno como cuidador & $\mathbf{N}$ & $\mathbf{\%}$ & & & 18,1 \\
Diurno & 51 & 61,4 & Noturno & & \\
Integral & 17 & 20,5 & & &
\end{tabular}

Cogitare Enferm. 2013 Out/Dez; 18(4):682-7 
profissionais de saúde, $75,9 \%$ informaram não realizar e $24,1 \%$ responderam que sim. Destes, $60 \%$ realizava tratamento com médicos; $20 \%$ com enfermeiras; $15 \%$ com agentes de saúde comunitária e 5\% fazem acompanhamento de saúde com nutricionista. Em relação à frequência com que consultam esses profissionais de saúde, $25 \%$ dos cuidadores responderam uma vez por mês, $15 \%$ duas vezes por mês; $10 \%$ trimestralmente, $10 \%$ quadrimestralmente e $40 \%$ referiram consultar o profissional semestralmente.

\section{DISCUSSÃO}

As mudanças que a DA determina na rotina familiar, o suporte social e situação financeira da família, grau educacional, estresse e fatores de risco como violência, desemprego e desarmonia familiar/marital devem ser considerados determinantes em relação ao cuidador deste tipo de paciente ${ }^{(5)}$. Neste sentido, esta investigação buscou ressaltar aspectos sobre qualidade de vida e formas de autocuidado de cuidadores de pacientes com DA, especialmente no que tange aspectos farmacoterapêuticos.

O papel de cuidador é visto, culturalmente, como uma função feminina ${ }^{(8-9)}$, incutindo à mulher do núcleo familiar o papel de tutora quando um adulto dependente necessite de cuidados. No presente estudo, a média de idade pode ser considerada baixa ( 38,9 anos) se comparado a outros estudos que relatam que os cuidadores pertencem à faixa etária superior a 55 anos $^{(10-12)}$. Tal diferença pode estar relacionada à profissionalização dos indivíduos participantes desta pesquisa, não pertencendo ao vínculo familiar do paciente, mas trabalhando profissionalmente com ele, uma vez que $90,4 \%$ dos entrevistados não possuíam relação de parentesco e $91,5 \%$ dos pacientes não coabitam com o mesmo.

A média de idade dos cuidadores aumenta com o agravamento da doença, mostrando que os cuidadores/ familiares são indivíduos em processo de envelhecimento, com necessidades específicas, peculiaridades e dificuldades, executando uma atividade que pode gerar desgaste e sobrecarga física e emocional, necessitando de cuidados específicos ${ }^{(13)}$.

Estudo revelou aumento na carga horária diária de trabalho entre cuidadores à medida que o paciente evoluía em relação à gravidade da demência, variando de três horas diárias, na demência leve, até mais de oito horas diárias quando o quadro atinge intensidade gra$\mathrm{ve}^{(13)}$. As cargas horárias citadas na presente pesquisa, seis e 12 horas, refletindo ser a prática do cuidado a principal atividade diária dos participantes do estudo.

A boa saúde física, envelhecimento saudável e boas condições financeiras são bons preditores de qualidade de vida. Aliado a isso, somam-se a presença de uma rede de apoio social, boa saúde mental e bem-estar espiritual ${ }^{(14)}$. Na presente pesquisa, o comportamento sedentário de mais da metade dos entrevistados $(56,7 \%)$ compromete fatores biológicos associados ao avançar da idade prejudicando a qualidade de vida dos cuidadores. Os principais fatores de risco para as doenças crônicas não transmissíveis, como hipertensão arterial são tabagismo, consumo excessivo de álcool, inatividade física, sobrepeso e obesidade, além do consumo inadequado de frutas e hortaliças. Nesta pesquisa o hábito de beber mostrou-se como frequente para um número considerável de cuidadores (18,1\%), o que pode interferir não apenas na sua própria saúde, mas também nas atividades de cuidado.

O sono é apontado pelos cuidadores como de grande dificuldade, mesmo que a maioria tenha mais de seis horas diárias $(83,2 \%)$, por ser interrompido muitas vezes por sobressaltos com o temor pela segurança dos pacientes. Torna-se uma alternativa, sempre que possível, o estímulo à atividade física do idoso durante o dia como uma maneira de manter o paciente ocupado e um estímulo para a mente e para o corpo ${ }^{(13)}$.

Cuidadores de pacientes com DA possuem maiores chances de ter sintomas psicológicos e psiquiátricos como depressão, ansiedade, insônia; maior frequência de conflitos familiares e problemas no trabalho; distúrbios físicos como hipertensão arterial, desordens digestivas e respiratórias e propensão a infecções ${ }^{(14)}$. O grau de cansaço do cuidador aumenta com a progressão da doença, bem como a sobrecarga de cuidado nas atividades do cotidiano, aliada a falta de informação em relação ao manejo dos idosos nestas atividades e a ausência de rede de suporte gera alto grau de estresse nos cuidadores $^{(13)}$. Esta situação gera principalmente ansiedade e disfunções musculares, citadas pelos entrevistados.

Alguns cuidadores relataram queixas de dificuldades auditivas e motoras atribuídas ao envelhecimento, o que pode ser motivo de preocupação pela responsabilidade que lhes é imputada no cuidado do paciente com DA. O grande desafio é lidar com o surgimento das doenças no cuidador, pelo fato de estarem envelhecendo, o que justifica a necessidade da colaboração de outras pessoas para manter o cuidado ${ }^{(15)}$. Além disso, a baixa frequência de busca por profissionais de saúde se configura em risco maior para o agravamento dos problemas de saúde, repercutindo na qualidade de vida 
dos cuidadores entrevistados. O uso de medicamentos mencionado pelos entrevistados corrobora os diagnósticos citados.

Os problemas de saúde do cuidador do paciente de DA muitas vezes são decorrentes das ansiedades do próprio cuidar. Entre os cuidadores, a depressão está intimamente relacionada com a solidão, à qualidade da relação entre o cuidador/idoso e a sua personalidade, cultura e nível de estresse. Nesta situação, torna-se fundamental a atenção dos profissionais de saúde na orientação e derivação aos serviços que possam lhe prestar atendimento e esclarecimento adequado, buscando melhora da qualidade de vida do cuidador e do idoso $^{(14)}$. Intervenções de suporte e aconselhamento fornecidas aos familiares/cuidadores dos pacientes com DA podem reduzir o nível de sintomas psiquiátricos existentes entre os familiares. A orientação farmacêutica pode proporcionar tanto benefícios ao paciente quanto ao cuidador, a medida que os benefícios do acompanhamento farmacoterapêutico atingem a ambos $^{(16)}$, reduzindo custos, melhorando as prescrições, promovendo maior adesão do paciente ao tratamento e controlando a possibilidade de reações adversas ${ }^{(17)}$.

Nota-se, pela análise dos resultados, a falta de identificação da doença com a orientação farmacêutica, instituindo-se um nicho de atuação para o profissional farmacêutico, que pode pautar suas atividades na melhoria da farmacoterapia, tendo em vista que o medicamento é um instrumento fundamental de recuperação e manutenção da saúde. A avaliação da farmacoterapia em idosos acometidos pela DA, bem como de seus cuidadores, é um importante instrumento de avaliação da qualidade da atenção prestada a estes grupos. Esforços para aprimorar a seleção, prescrição, a dispensação e a utilização de fármacos devem constituir prioridade nos programas de atenção ao idoso ${ }^{(18)}$. Quando há dificuldade em utilização de medicamentos, a educação em saúde deve enfocar a adesão como uma relação colaborativa e acima de tudo de corresponsabilidade, por meio de troca de experiências, questionamentos e humanização dos pacientes, e não em atos dominadores ${ }^{(19)}$.

Partindo das informações fornecidas pelos cuidadores, observa-se que esses precisam implementar ações que amenizem o estresse e previnam desgaste físico e emocional no trato com os portadores de DA. Manter contato com outras pessoas que também se ocupam da função de cuidador de pacientes acometido da doença, podem contribuir com o resgate social do cuidador, proporcionando momentos de troca de experiências e desabafos que amenizam sua vida ${ }^{(5)}$.
O familiar de paciente com DA ou ainda o profissional que pretende trabalhar como cuidador deverá ter entre suas habilidades e conhecimentos a familiaridade com os transtornos comuns do envelhecimento e com suas possibilidades de tratamento; informações sobre diagnóstico e avaliações dos transtornos do envelhecimento; conhecimento sobre as disponibilidades dos serviços de saúde para o idoso. Também são necessárias habilidades clínicas que permitam tanto prover orientação relativa ao comportamento do idoso, como aquelas que permitam levar o cuidador a identificar sentimentos e comportamentos relativos ao idoso, compreendê-los e/ou modificá-los, de modo a resultar em melhora de sua qualidade de vida, e de sua relação com o idoso, com outros familiares e com pessoas do seu convívio ${ }^{(20)}$.

\section{CONSIDERAÇÕES FINAIS}

O estudo buscou registrar a importância da atenção em saúde, em especial a atenção farmacêutica ao cuidador de paciente acometido da DA, por se tratar de um processo que envolve a sobrecarga e o impacto que a tarefa de cuidar provoca na vida do cuidador. Como a pessoa acometida por DA necessita de dedicação exclusiva, isso, muitas vezes, faz com que o cuidador deixe de realizar atividades pessoais em favor dos cuidados dispensados ao paciente, o que pode induzi-lo ao isolamento social e a depressão.

Dentre os aspectos investigados, que podem prejudicar a qualidade de vida dos cuidadores, estão o ato de esquecer-se de tomar um medicamento necessário ao seu bem-estar, inatividade física, poucos cuidados com a saúde pessoal, incluindo-se a baixa frequência de consultas médicas e exames de rotina, exigindo tempo, recursos financeiros, entre outros. Estas situações despertam a necessidade de avaliar, compreender e contribuir com propostas que possibilitem uma melhor qualidade de vida aos cuidadores de pacientes com DA. Neste contexto, a atenção farmacêutica ao cuidador deve estar direcionada a desenvolver ações e/ou intervenções que preencham as necessidades observadas, favorecendo a qualidade de vida e contribuindo com a promoção da saúde.

\section{REFERÊNCIAS}

1. Almeida KS, Leite MT, Hildebrandt, LM. Cuidadores familiares de pessoas portadoras de Doença de Alzheimer: revisão da literatura. Rev. Eletr. Enf. [Internet] 2009;11(2):403-12. 
2. Tiedman M, Kim EC, Flurie R, Black KK, Brandt NB. Doença de Alzheimer: opções de tratamento actuais e desenvolvimentos futures. Patient Care. 2011;12:44-59.

3. Verdullas RA, Ferreira M, Nogueira VO. Dificuldades enfrentadas pelo cuidador familiar mediante o paciente com mal de Alzheimer em fase avançada. Saúde colet. 2011;50(8):109-13.

4. Campos EP. Quem cuida do cuidador: uma proposta para profissionais da saúde. Petrópolis, RJ: Vozes; 2011. $150 \mathrm{p}$.

5. Almeida KS, Leite MT, Hildebrandt LM. Cuidadores familiares de pessoas portadoras de Doença de Alzheimer: revisão da literatura. Rev. eletrônica enferm, 2009; 11(2):403-12.

6. Gil AC. Como elaborar projeto de pesquisa. $4^{\mathrm{a}}$ ed. São Paulo: Atlas; 2009.

7. Biernarcki P, Waldorf D. Snowball sampling-problems and techniques of chain referral sampling. Sociological Methods and Research. 1981;10:141-63.

8. Cruz MN, Hamdan AC. O impacto da doença de Alzheimer no cuidador. Psicol. estud. Maringá. 2008;13(2):223-9.

9. Kochhann R, Borbal E, Cerveiral MO, Onyszko D, Jesus A, Forster L, et al. Neuropsychiatric symptoms as the main determinant of caregiver burden in Alzheimer's disease. Dement Neuropsychol. 2011;5(3):203-8

10. Lenardt MH, Hautsch WM, Seima MD, Pereira LF. A condição de saúde e satisfação com a vida do cuidador familiar de idoso com Alzheimer. Colomb. Med. 2011;42(2):17-25.

11. Magalhaes SB, Franco ALS. Experiência de profissionais e familiares de pacientes em cuidados paliativos. Arq. bras. psicol. 2011;64(3):94-109

12. Pinto MF, Barbosa DA, Ferreti CEL, Souza LF, Fram DS, Belasco AGS. Qualidade de vida de cuidadores de idosos com doença de Alzheimer. Acta Paul. Enferm. 2009;22(5):652-7.

13. Novelli MMPC, Nitrini R, Caramelli P. Cuidadores de idosos com demência: perfil sociodemográfico e impacto diário. Rev. Ter. Ocup. Univ. São Paulo. 2010;21(2):139-47 .

14. Paula JA, Roque FP, Araujo FS. Qualidade de vida em cuidadores de idosos portadores de demência de Alzheimer. J. bras. psiquiatr. 2008;57(4):283-7.
15. Gaioli CCLO, Furegato ARF, Santos JLF. Perfil de cuidadores de idosos com doença de Alzheimer associado à resiliência. Texto Contexto Enferm. Florianópolis. 2012;21(1):150-7.

16. Menezes Ferreira MM, Rabinovich, EP. Família do idoso com doença de Alzheimer: um estudo de caso. Saúde colet. 2012;55(9):7-12.

17. Medeiros EFF, Moraes CF, Karnikowski M, Nóbrega OT, Karnikowski MGO. Intervenção interdisciplinar enquanto estratégia para o Uso Racional de Medicamentos em idosos. Ciênc. saude colet. [Internet]. 2011;16(7):3139-49.

18. Ribeiro AQ. Qualidade do uso de medicamentos por idosos: uma revisão dos métodos de avaliação disponíveis. Ciênc. saude colet. 2005;10(4):1037-45.

19. Lyra Jr DP. A farmacoterapia no idoso: revisão sobre a abordagem multiprofissional no controle da hipertensão arterial sistêmica. Rev. Latino-Am. Enfermagem. 2006;4(3):435-41. 20 Inouye K, Pedrazzani ES, Pavarini SCI. Implicações da doença de Alzheimer na qualidade de vida do cuidador: um estudo comparativo. Cad. Saúde Pública . 2010;26(5):891-9. 